\title{
PNEUMATOSIS INTESTINALIS IN CHINESE CHILDREN
}

\author{
BY \\ C. S. MUIR \\ From the Department of Pathology, University of Malaya
}

(RECEIVED FOR PUBLICATION MARCH 5, 1958)

Pneumatosis cystoides intestinorum hominis is a rare condition. Although about 250 cases have been described to date only seven of these have come from the Orient: five Chinese adults (Tung and Ngai, 1933; Yin, 1937; Ling, Ku and Su, 1948), and two Indian adults (Tribedi, 1941; Davies, 1941). This paper records five cases occurring in Singapore, one in an adult, the remainder in children.

The lesion consists of gas-containing cysts of varying size, either sessile or pedunculated, within the wall of the intestinal tract. There may, in addition, be similar cysts on the surface of other abdominal organs and on the parietal peritoneum.

\section{Case Histories}

Case 1. C.J.M., a 3-month-old male Cantonese child, was admitted to the General Hospital, Singapore, on August 20, 1953, and died five minutes after reaching the ward. The child's mother stated that he had had diarrhoea for the past two days. Over a similar period of time she had noticed increasing breathlessness. The child's temperature was $104^{\circ} \mathrm{F}$. Dyspnoea was extreme and numerous crepitations were heard on auscultation of the lung bases.

Dr. E. B. La'Brooy, who performed an autopsy, reported that the body was that of a thin, male Chinese infant, height $2 \mathrm{ft}$. 4 in., weight 1 st. $1 \mathrm{lb}$. The heart appeared normal. The respiratory passages were partially blocked by mucopurulent exudate, and the lungs showed patchy areas of consolidation with areas of collapse posteriorly. The liver showed gross fatty change. Hyperaemia of the mucosa was present in the small intestine. Two lymph follicles showed superficial necrosis and bile staining of sloughing tissue. The large intestine showed several irregular ulcers of lymph follicles. There were numerous bubbles of gas in the mucosa of the ileum. The stools were not cultured.

Histology. A section of colon showed well-marked pneumatosis. The majority of gas spaces had no lining endothelium (Fig. 1, top left). Several of these unlined spaces had giant cells in the wall and an occasional macrophage round about. Another space showed an incipient lining, which appeared to be formed by condensation of surrounding tissue. In this wall (Fig. 2), three giant cells were seen (cf. Fig. 30: Wright, 1930). Moderate numbers of macrophages were present in the neighbourhood.

\section{$<$}

Case 2. A.B.H., aged 1 month, a male Chinese infant, was admitted to the General Hospital, Singapore, in a dehydrated condition, with a three-day history of diarrhoea and vomiting after feeds. No pathogenic organisms were grown from the stools. The child died.

Dr. Ng Chiau Gian found a patent foramen ovale on autopsy. There was a thin layer of sero-purulent fluid inside the peritoneal cavity, and small mucosal ulcers in the lower part of the ileum and colon. In a portion of the ileal wall, which showed no signs of inflammation, there were numerous blebs of gas, possibly post-mortem in origin.

Histology. Sections of colon showed a very welldeveloped pneumatosis. The gas spaces were lined by a low endothelium. Towards the centre of the section an abscess containing mononuclears, polymorphs and much debris was present. This extended from the mucosa to the muscle layers and was of the 'collar-stud' type (Fig. 3). Just below this abscess, within the muscle layers, an amoeba was seen (Fig. 4).

Case 3. G.H.J., a 1-month-old male Hokkien child, was admitted to the General Hospital, Singapore, on December 1, 1956, with a complaint of persistent regurgitation of feeds of several days' duration. A diagnosis of duodenal stenosis was made and on December 15 laparotomy was performed. A volvulus of mid-ileum was reduced and numerous adhesions divided. The child died on January 2, 1957, after a short period of vomiting.

At autopsy the body was that of a thin, male Chinese infant. The abdomen was rather bloated, and there was a well-healed transverse lower abdominal surgical scar. The heart appeared normal. The lung parenchyma showed early bronchopneumonic change. The normal anatomy of the gut was deranged by multiple adhesions: a major portion of caecum was tacked down to the liver and the appendix was adherent to the small bowel. The loops of small bowel were adherent to each other, showing numerous kinks although no definite obstruction 


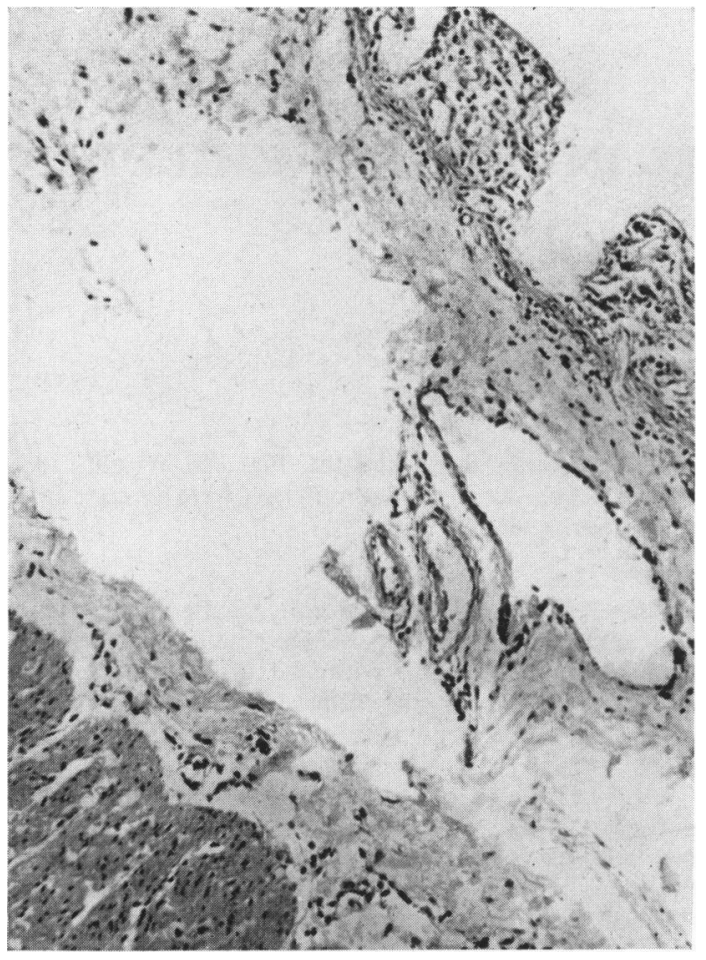

FIG. 1.-P.M. 1492/53. Section of intestinal wall showing, at middle right, a gas space with an incipient lining. On either side is unlined gas space. H. and E. $\times 135$.

could be detected. One portion of jejunum was tacked down between the liver and the diaphragm. The gut was generally dilated, red in colour and in places showed desquamation of the mucosal epithelium.

Histology. Section of the ileum showed pneumatosis (Figs. 5, 6 and 7). Nearly all the gas spaces had an endothelial lining although this was not well marked in places. Macrophages were moderately plentiful; the cytoplasm was less bulky than in other sections. Moderate numbers of giant cells were seen (Fig. 8).

Case 4. O.B.C., a 63-year-old Henghwa male, was admitted to the General Hospital, Singapore, on April 25, 1957, with a complaint of four months' increasing abdominal swelling. A firm nodular mass was palpated in the upper abdomen and the liver was grossly enlarged. He was considered to be suffering from gastric carcinoma.

Dr. A. O. Aaron's autopsy report stated that the heart appeared to be normal. The lungs were the seat of numerous small circumscribed tumour nodules. The liver weighed 4,300 g., being largely replaced by secondary tumour. There was no evidence of cirrhosis. There was a large circular carcinomatous ulcer $5 \mathrm{~cm}$. in diameter on the lesser curvature of the stomach close to the pylorus. The submucosa of the ileum and colon showed pneumatosis.

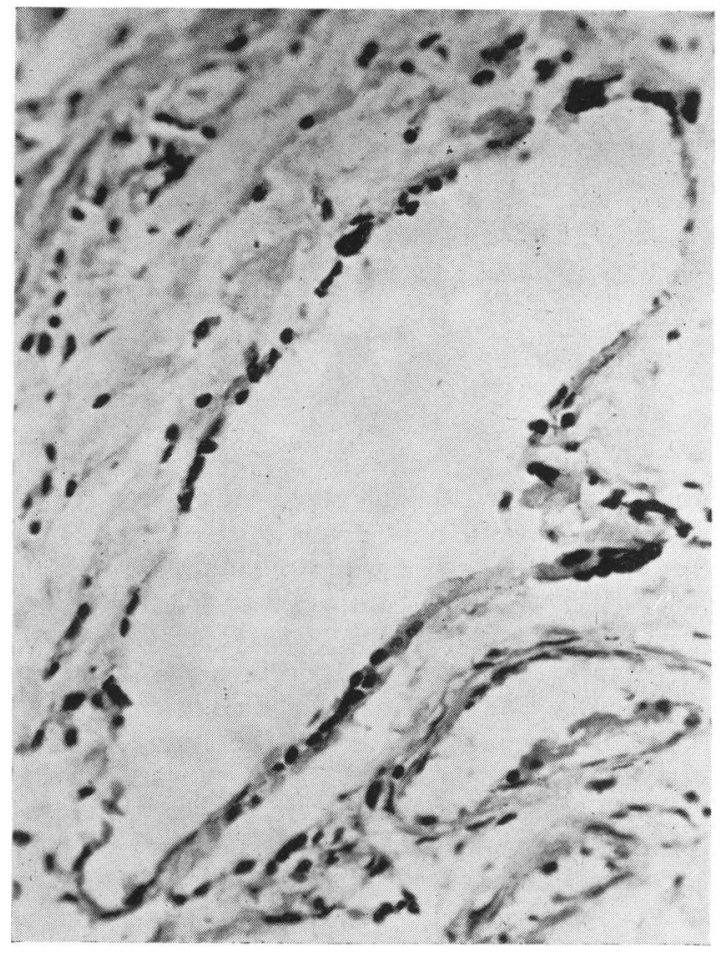

FIG. 2.-P.M. 1492/53. Higher magnification of the gas space above shows it to be partially lined by a condensation of surrounding tissue. In the wall three giant cells are seen. Moderate numbers of macrophages are present in the surrounding tissue. H. and E. $\times 336$.

Histology. Section of the colon showed moderately well-developed pneumatosis. All the gas spaces were lined by a low endothelium. Very few giant cells or macrophages were seen.

Case 5. N.P.M., a 12-year-old Hokkien girl, was admitted to the General Hospital, Singapore, on December 26, 1957, and died one hour later. She gave a history of two days' abdominal pain associated with vomiting. She had passed numerous stools on the day prior to admission.

At autopsy the heart appeared normal. The lungs showed changes, possibly early bronchopneumonic. The entire gut from mid-jejunum to descending colon was inflamed and showed areas of patchy superficial necrosis. The necrotic areas were deeply bile stained. In the mid-jejunum and upper ileum several small areas, each about $5 \mathrm{~cm}$. long, were seen which showed air bubbles in the gut wall. This was probably early pneumatosis.

Histology. Section of the lower jejunum (Fig. 9), showed a moderate degree of disruption of the tissues of the sub-mucosa. This was presumably caused by gas. Around these areas macrophages and eosinophils were prominent (Figs. 10 and 11; cf. Fig. 25: Wright, 1930). These macrophages had a prominent ovaloid nucleus and a bulky homogeneous pink cytoplasm. 


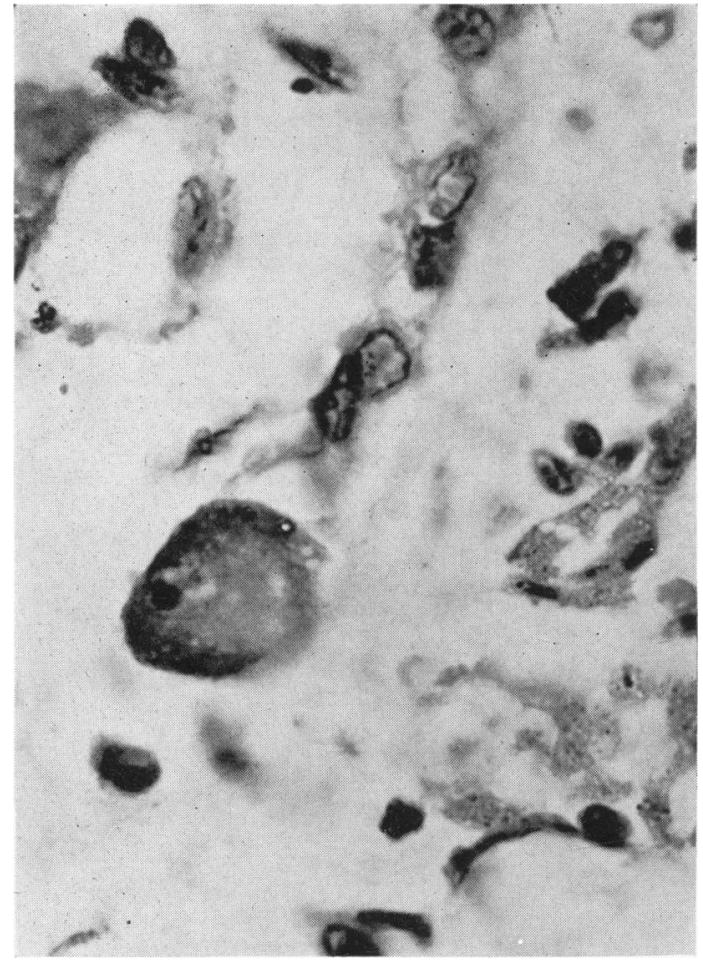

FIG. 3.-P.M. 1167/57. Amoebic abscess of ascending colon superimposed on pneumatosis. H. and E. $\times 135$.

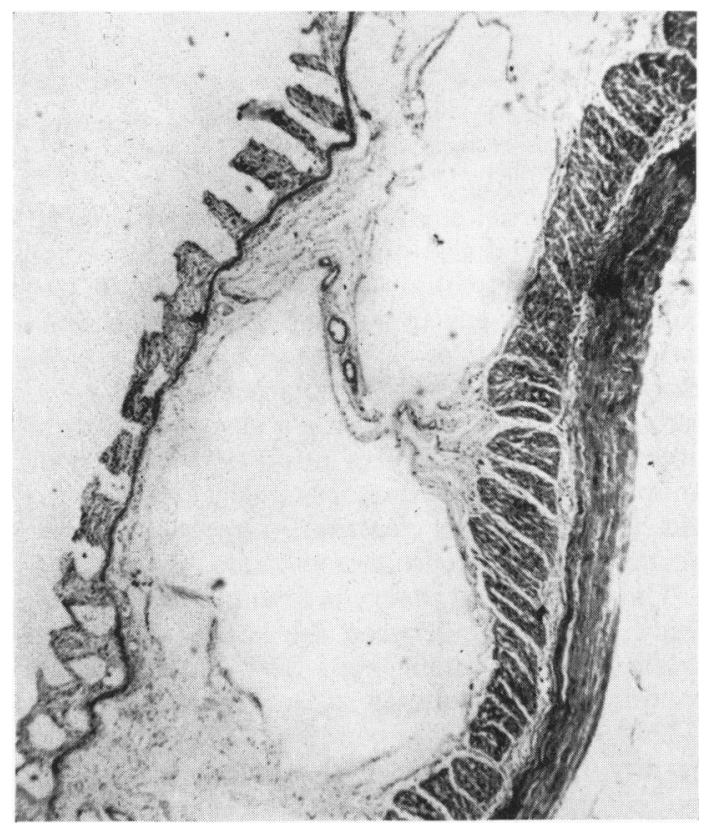

FIG. 5.-P.M. 7/57. Section showing submucosal pneumatosis.

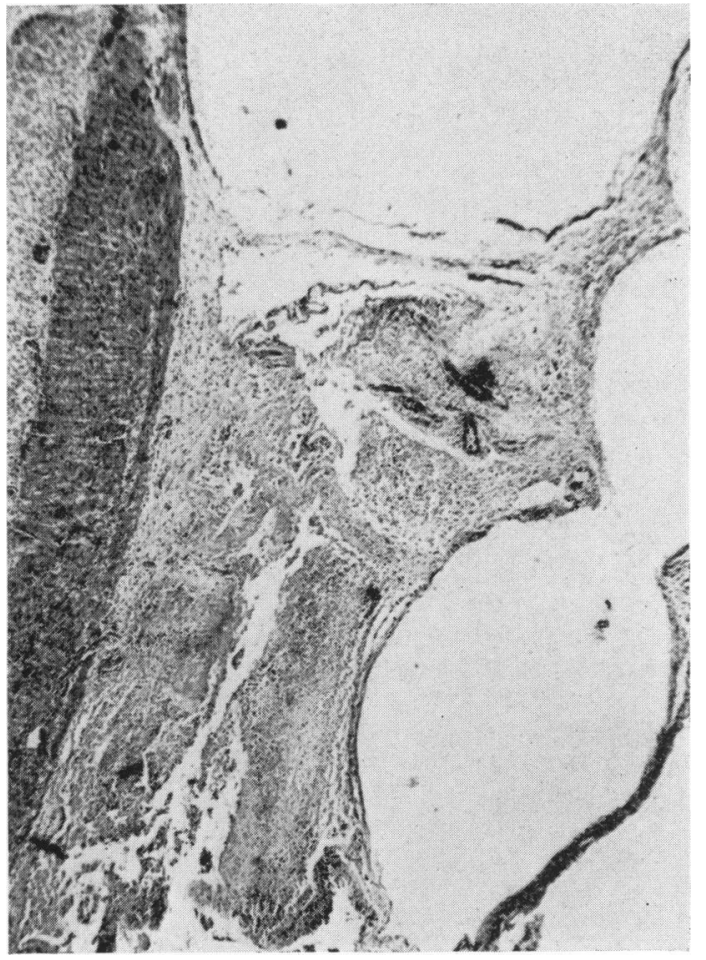

FIG. 4.-P.M. 1167/57. An amoeba within the muscle coats' H. and E. $\times 1,200$.

Elsewhere in the section several lined gas spaces were seen. One of these (Fig. 12), was lined for about threequarters of its circumference by a low endothelium, the remainder by a condensation of surrounding tissue. Around this space moderate numbers of macrophages were present.

\section{Aetiology}

The exact cause of pneumatosis is unknown. Several theories have each, from time to time, attracted some support.

First in the field was the neoplastic hypothesis. Bang (1876) described the first well-documented case and considered the cysts to be the product of a new growth, which, taking its origin from giant cells in the lymphatic vessels, spread, elaborating a gas as it did so. Mair (1907) believed that the lesion was a true neoplasm whose cells produced gas. Finney (1908) who reported the first American case subscribed to this viewpoint.

The bacterial theory attracted workers who considered that the cysts were the work of gas-producing organisms. Dupraz (1897) did, indeed, isolate a variety of organisms which he termed Bacterium 


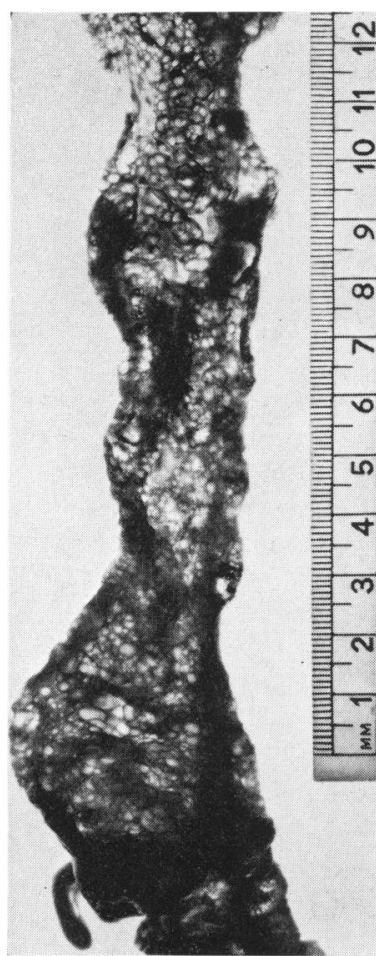

FIG. 6.-P.M. 7/57. Macroscopic appearance of caecum by incident light.

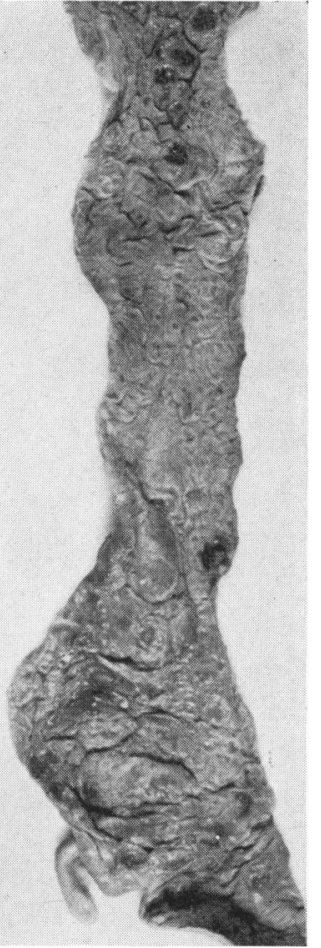

Fig. 7.-Same portion of gut as in Fig. 6. By transmitted light the extent of involvement is much more readily appreciated.

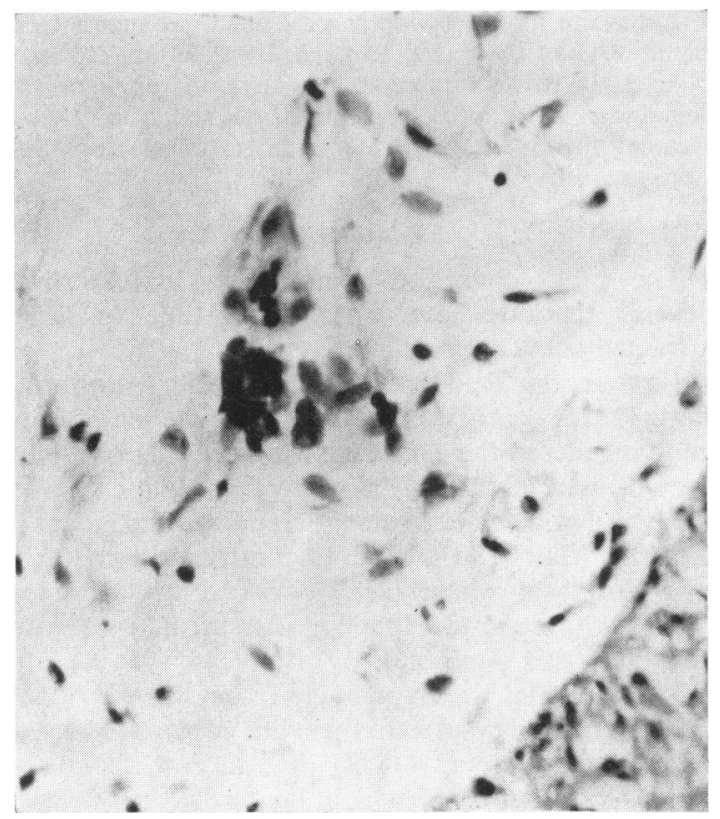

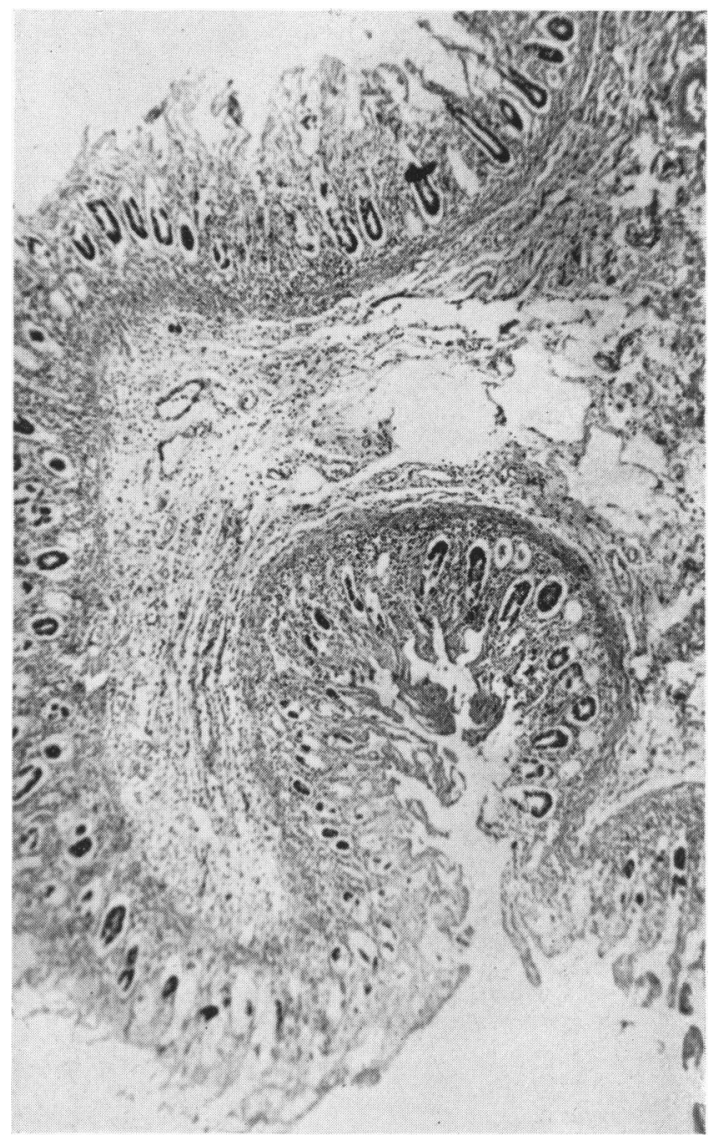

FIG. 9.-P.M. 2609/57. The lower jejunum shows a moderate degree of disruption of the sub-mucosal tissues. H. and E. $\times 37$.

coli lymphaticum aerogenes which, if given orally to animals, reproduced the lesions. All his animals died, however, within 48 hours. Naeslund (1924) cultured from gas cysts in the intestinal wall of swine a variant of Esch. coli which he termed Bacillus pneumatosis. He obtained weakly positive serological reactions between a suspension of these organisms and the sera of positive swine. Healthy animals, i.e., those without gas cysts of the intestines, did not show this reaction. Injection of these bacteria failed to reproduce the lesions.

The biochemical theory is favoured by those who draw an analogy between the lesions in man and those occurring in the pig. Swine suffer from the condition quite frequently, a fact known since Mayer (1825) described the condition in an otherwise healthy hog. Known as swine emphysema it is

FIG. 8.-P.M. 7/57. A giant cell in the wall of a partially lined gas space. H. and E. $\times 336$. 


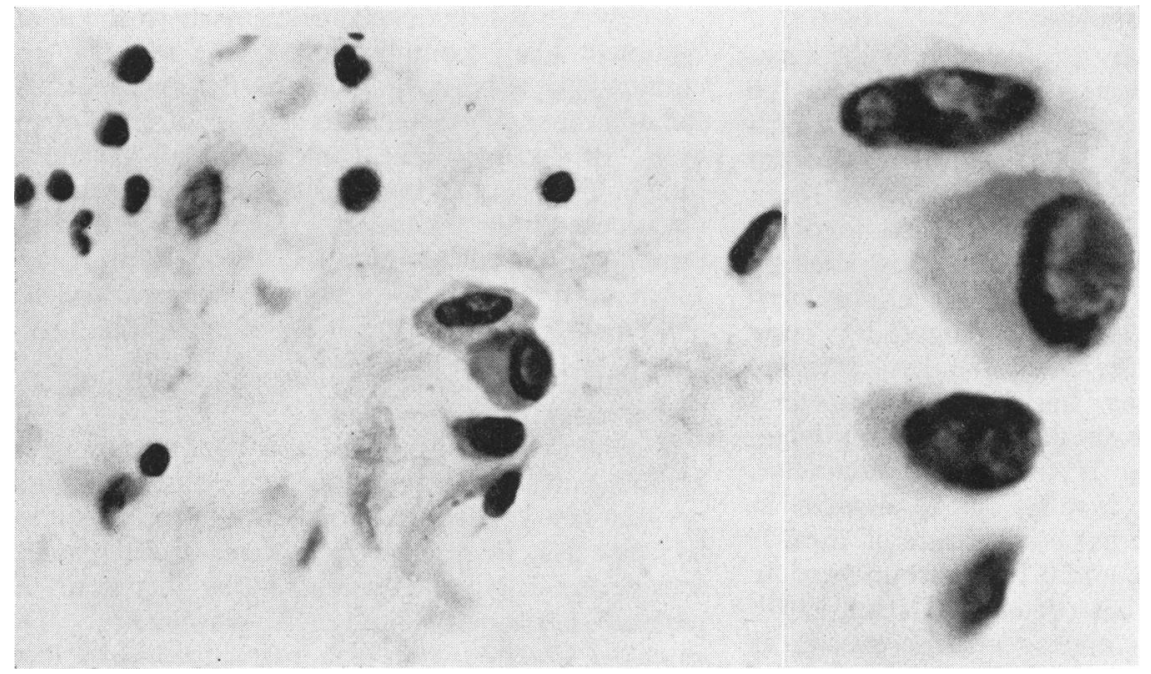

FIG, 10.-P.M. 2609/57. Macrophages around the gas spaces in the intestinal wall. H. and E. $\times 880$.

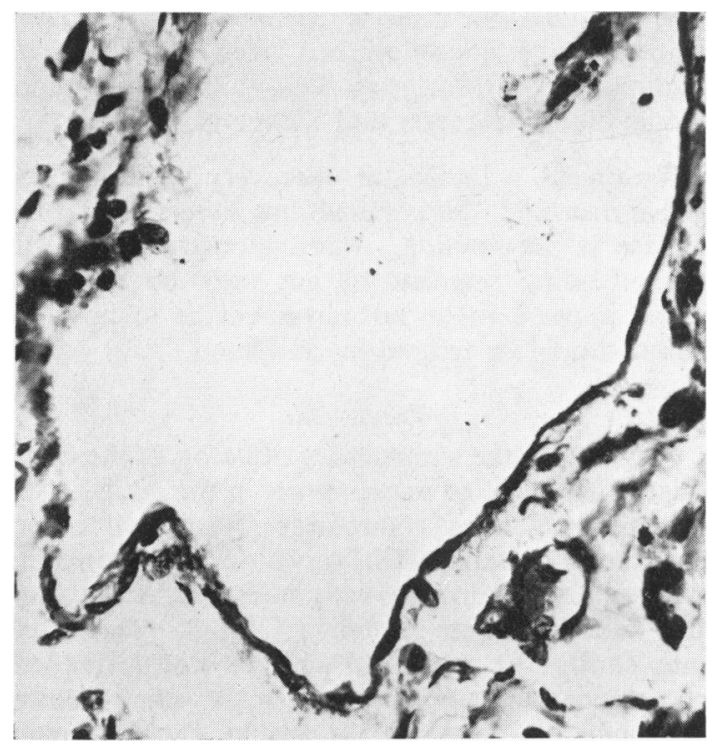

Fig. 12.-P.M. 2609/57. A low endothelium partially lines this gas space on the bottom and right sides, blending gradually at the top left into a condensation of the surrounding tissues. Moderate numbers of macrophages are present. H. and E. $\times 350$.

related in some way to diet, as the disease can be readily reproduced on feeding a diet of polished rice (Biester, Eveleth and Yamoshiro, 1936). Such a relationship is not apparent in man. Pneumatosis has also been observed in sheep (Mills, 1925) and in chickens (Naeslund, 1924).

At present the mechanical theory seems to attract the most adherents. If air be injected directly into the gut wall it follows the lymphatics to the mesentery and within a few days is gradually absorbed. Masson (1925) concluded that lymph stagnation is the basis of the phenomenon. This occurs in tortuous or obstructed lymph vessels, carbon dioxide and lactic acid being absorbed into the obstructed segment. Carbon dioxide is liberated and nitrogen, oxygen and methane diffuse in, the relative proportions of the gases being governed by their partial pressures.

Tung and Ngai (1933) expended considerable effort in trying to reproduce pneumatosis in the dog. They attempted to mimic as closely as possible the conditions obtaining in their patients, both of whom had, in addition to pneumatosis, stenosing peptic ulcers. They reproduced such a stenosing ulcer in one of their dogs and filled the stomach with air and salt solution. Air bubbles appeared, both inside and outside the lymphatics, which moved distally with each peristaltic movement. The animals were allowed to recover from anaesthesia. On the second day after operation the dogs vomited, refused to eat, and appeared quite sick. On reopening the abdomen air bubbles were seen in the lymphatics of the gastric serosa and in those of the omental attachments. Straus (1954) attempted to do the same in the large intestine of the dog. He found that gas cysts did form slowly if air were injected into the lumen of a segment of bowel which had been obstructed both distally and proximally. It seems, therefore, that obstruction favours the formation of these cysts, although it cannot be the whole answer as one would expect to see them more frequently in association with, say, obstructing carcinomata of the colon. 


\section{Pathology}

The ileum, followed by the colon, are the most frequently affected portions of the gut; both may be involved at once (MacKenzie, 1951). The stomach and gall bladder (Mills, 1925) and the falciform ligament of the liver (Pybus, 1934) have been reported as affected. The amount of gut involved varies. It is often confined to a loop constricted by adhesions or volvulus, sometimes to a loop containing intestinal parasites, usually nematodes (Tung and Ngai, 1933). In the adult the gas cysts are found mainly in the serosa. In children the cysts tend to be submucosal, cysts in the muscle coats being rare. The average cyst is $4-5 \mathrm{~mm}$. in diameter. Koss (1952) reports one cyst $3.5 \mathrm{~cm}$. in diameter in the distal caecum. The gas is a mixture of $70-90 \%$ nitrogen, $3-20 \%$ oxygen, and $0-15 \%$ carbon dioxide. Traces of methane have been detected (Urban, 1910).

In children the cysts appear microscopically as thin-walled sacs lined entirely, or in part, by a single layer of flat endothelium. This lining may not be present. In adults as a rule giant cells are present; in the child they may not be so conspicuous. Wright (1930) has shown that air acts as a foreign body evoking a giant cell response. These giant cells are in many instances mere aggregations of nuclei some 10 to 50 in number and are found near the gas-filled space (Fig. 8). Tung and Ngai (1933) describe in their adult cases a second type of cyst lined by a dense fibrous tissue. In their Fig. 6 they show giant cells in the lumen of one example of this type of cyst, which they regard as being older than the thin-walled variety. They made serial sections of their specimens and found that the endothelium lined spaces communicated with lymphatic vessels, whereas the fibrous variety did not. Koss (1952), examining serial sections, was unable to demonstrate a breach of the continuity of mucosa in the region affected by gas cysts or to demonstrate a definite transition between a cyst and a lymphatic vessel. It seems, therefore, that in time the thin-walled cysts become fibrosed and obliterated. The photomicrographs in his paper show a similar disposition of the giant cells in thick-walled cysts.

\section{Clinical Features}

In the infant the most common presenting symptom is diarrhoea (Botsford and Krakower, 1938; Judge, Cassidy and Rice, 1949; MacKenzie, 1951). In the adult pyloric obstruction following peptic ulcer is found in about $60 \%$ of cases (Nitch and Shattock, 1919). Pyloric lesions tend to be associated with pneumatosis of the small gut, while obstructive signs are found more commonly with large gut lesions (Kreeger and Littmann, 1954).
Intestinal obstruction is, particularly in the adult, the most likely complication. This may be precipitated by volvulus induced by the cyst mass, by the adhesions which seem to arise as a result of the cysts, or by mechanical blockage of the bowel lumen. Koss (1952) quotes a case in which pneumatosis caused intussusception. Haemorrhage from the bowel may occur (Matthews, 1954; Kreeger and Littmann, 1954; Pontius, 1954). Pneumoperitoneum will result if a sufficient number of cysts burst.

Diagnosis. Diagnosis is usually made on the operating table or at autopsy.

A straight radiograph of the abdomen may be helpful, particularly so if there should be a pneumoperitoneum. Pneumoperitoneum may be simulated by the gas from the mesenteric cysts passing retroperitoneally and accumulating between the parietal peritoneum and the diaphragm. The so-called Chilaiditi sign (the interposition of a loop of bowel between liver and diaphragm) is often positive. Barium meal or enema will show filling defects. The condition has been diagnosed on sigmoidoscopy (Matthews, 1954) and on sigmoidoscopic biopsy (Kreeger and Littmann, 1954).

Treatment. Incidental discovery calls for no active measure. Koss (1952) has observed that the disease is self-limiting. Should obstruction occur the offending segment of gut may be removed. There appears to be no agreement as to how the lesion should be treated in childhood.

\section{Discussion}

Apart from the submucosal situation of the cysts, Case 4 is typical of pneumatosis in the adult. The rest show features common to nearly all others described in infants. Diarrhoea was the presenting symptom. A volvulus with intestinal obstruction and adhesions were found in Case 3. This latter case shows the Chilaiditi sign, a not infrequent occurrence in pneumatosis with small bowel involvement. Koss (1952) would exclude many of the cases reported in children, preferring to class them as intestinal emphysema. He observes that in many of these cases on microscopy, the cyst wall is devoid of epithelial lining, and cites the cases of Botsford and Krakower (1938) and Judge et al. (1949). He states, 'The cases in which there is evidence of cyst lining are accepted, and the others are considered as cases of emphysema, possibly a terminal event, with intestinal gas penetrating into the intestinal wall generally and not selectively into the lymphatics.'

While one must respect the views of the author of a careful and exhaustive review, he and many 
others have failed to appreciate the significance of the work of Wright (1930). Wright injected, under aseptic conditions, various gases into the subcutaneous tissues of guinea pigs. He found that nitrogen remained in the tissues for three days after injection and at the end of this time the crepitation typical of surgical emphysema could still be elicited. With oxygen, a gas which diffuses much more readily, crepitation could be detected at the end of 24 hours.

A study of the cellular response to these gases showed that while the response varied quantitatively with each gas used, qualitatively it was much the same. Within two days of the introduction of gas into the subcutaneous tissues, tissues which are surely not so different from those of the sub-mucosa or sub-serosa, there appeared round the gas spaces epithelioid type cells, derived, he felt, from the monocytes, by virtue of their appearance when stained supravitally. By the tenth day numerous giant cells derived from these epithelioid-like cells were present, some being $15-40 \mu$ in diameter. By the fourth day portions of the walls of the gas spaces were lined by flat cells, which morphologically resembled an endothelioid or serous lining. Although he thought that the possibility that these cells arose from capillary or lymphatic endothelium could not be ruled out, he could find no evidence that they did so and concluded that this lining arose from the epithelioid cells. By the end of two months the walls of these gas spaces were thickened and the lumina contained many giant cells, a picture closely resembling the 'older' cysts of Tung and Ngai (1933).

Although the author has been unable to identify the epithelioid nature of the cells described by Wright (1930), nearly all the sections examined from the cases of pneumatosis described showed a characteristic cell which has been described as a macrophage in the text. This cell, with an oval or round nucleus and a homogeneous dusky pink cytoplasm, is found in great numbers around early lesions and to a lesser extent around the more advanced ones. This cell may be the intestinal counterpart of Wright's epithelioid cell, and, indeed, cells which are described as mononuclears in Fig. 25 of his paper look identical with the author's macrophages.

The sequence of events would thus seem to be:

(a) The intestinal gases gain entrance to the sub-mucosal tissues, disrupting them to form gas spaces (Fig. 9) around which a macrophage response is evoked (Fig. 10).

(b) The tissues surrounding these spaces condense to form a lining, which gradually becomes altered to an endothelium (Figs. 2 and 12). In these walls typical giant cells, probably derived from the macrophages, are found (Figs. 2 and 8 ).

(c) As the gas space, now a lined gas cyst, becomes older, it may either dilate as in Figs. 5 and 6 or sclerose as in the cases of Tung and Ngai (1933).

This histological interpretation is open to criticism. It does not explain the mode of formation of sub-serosal gas cysts, nor the mode of entry of the gases when there is apparently no breach in the gut wall. It may be that there are two mechanisms of genesis for pneumatosis, a lymphatic one, and the more mechanical one outlined above. It is felt that the exclusion of certain cases by Koss (1952) is, on the basis of the evidence presented above, unwarranted. The immunological approach of Naeslund (1924) is worth repeating.

It is not without interest to note that even in the fresh specimen I could not elicit the crepitation which is so characteristic of subcutaneous emphysema. Although this absence of crepitus has not been commented on before, no reference to its presence could be found in other cases.

\section{Summary}

Five cases of pneumatosis occurring in persons of Chinese race are reported. The literature is briefly reviewed. Special reference is made to the differences of opinion which exist on the classification of gas cysts of the intestine in children, and a theory of pathogenesis for the condition is promulgated.

I wish to thank Professor Kirk for kind help and criticism; Mr. V. Nalpon for sections and photomicrographs and my wife for secretarial assistance.

Bang, B. L. F. (1876). Nord. med. Ark., 8, 1. (Cited by Finney (1908). Loc. cit.)

Biester, H. E., Eve!eth, D. F. and Yamashiro, Y. (1936). J. Amer. yet. med. Ass., 88, 714 .

Botsford, T. W. and Krakower, C. (1938). J. Pediat., 13, 185.

Davies, S. T. (1941). Indian med. Gaz., 76, 94 .

Dupraz, A. L. (1897). Arch. Méd. exp., 9, 282.

Finney, J. M. T. (1908). J. Amer. med. Ass., 51, 1291.

Judge, D. J., Cassidy, J. E. and Rice, E. C. (1949). Arch. Path. (Chicago), 48, 206.

Koss, L. G. (1952). A.M.A. Arch. Path., 53, 523.

Kreeger, N. and Littman, L. (1954). New Engl. J. Med., 251, 779.

Ling, C. Y.. Koo, S. N. and Su, Y. H. (1948). Chin. med. J., 66, 435.

MacKenzie, E. P. (1951). Pediatrics, 7, 537.

Mair, W. (1908). Med. Chron., 47, 422.

Masson, P. (1925). Ann. Anat. path., 2, 541.

Matthews, F. J. C. (1954). Brit. med.J., 1, 851.

Mayer, J. (1825). Quoted by Koss (1952). I.oc. cit.

Mills, H. W. (1925). Surg. Gynec. Obstet., 40, 387.

Naeslund, J. (1924). Zur Kenntnis der Pneumotosis cystoides

intestinorum. Thesis. Uppsala, Stockholm. Sect. of Path., 12, 46.

Olson, J. D. (1954). A.M.A. Arch. Surg., 68, 899.

Pontius, G. V. (1954). In discussion on paper by Olson (1954). Loc. cit.

Pybus, F. C. (1934). Brit. J. Surg., 21, 539.

Straus, F. H. (1954). In discussion on paper by Olson (1954). Loc. cit.

Tribedi, B. P. (1941). Calcutta med. J., 38, 285.

Tung, P. C. and Ngai, S. K. (1933). Chinese med. J., 47, 1.

Urban, K. (1910). Wein. med. Wschr., 60, 1750

Wright, A. W. (1930). Amer. J. Path., 687.

Yin, Y. C. (1937). Chinese med. J., 51, 541. 